\title{
Endoparasitic Arthropods
}

\author{
El Awad Mohamed El Hassan
}

\subsection{Linguatula serrata Infection in Camels (Halzoon; Marara Syndrome)}

Linguatula serrata, the nasal worm of canids, is a zoonotic pentastome that uses dogs and other carnivores as definitive hosts while its larval stages are found in the mesenteric and mediastinal lymph nodes, liver, and lungs of herbivores, e.g., camels, sheep, goats, and cattle, as well in humans (Sazmand \& Joachim, 2017). Human infection with $L$. serrata nymphs causes an intense hypersensitivity reaction in the upper respiratory tract and the buccopharyngeal mucosa. The condition is known as Halazoun in Syria, Lebanon, and other parts of the Middle East. In Sudan, it is known as the Marara syndrome-Marrara is a popular dish in Sudan, consisting of raw liver, lungs, trachea, and rumen of camels, goats, and sheep, which could be infected with larvae of L. serrata. Marara is typically consumed during festivals.

\section{Reference}

Sazmand, A., \& Joachim, A. (2017). Parasitic diseases of camels in Iran (1931-2017) - A literature review. Parasite, 24, 1-15. 